\title{
Maternal and Perinatal Outcomes of Pregnancies Complicated with Pre- gestational and Gestational Diabetes Mellitus in Saudi Arabia
}

Hayfaa A Wahabi ${ }^{1}$, Amel Fayed ${ }^{2}$ and Samia A Esmaeil ${ }^{1}$

${ }^{1}$ Sheikh Bahmdan Chair of Evidence-Based Healthcare and Knowledge Translation, College of Medicine, King Saud University, Riyadh, Saudi Arabia

${ }^{2}$ Department of Basic Sciences, Division of Biostatistics, College of Medicine, King Saud Ben Abdel Aziz, University for Health Sciences, Riyadh, Saudi Arabia

"Corresponding author: Hayfaa A Wahabi, Associate Professor, Department of Basic Sciences, Division of Biostatistics, College of Medicine, King Saud University Riyadh, Central Saudi Arabia, Tel: +966553294955; E-mail: umlena@yahoo.com

Received: May 19, 2014, Accepted: June 27, 2014, Published: July 04, 2014

Copyright: (c) 2014 Wahabi HA, et al. This is an open-access article distributed under the terms of the Creative Commons Attribution License, which permits unrestricted use, distribution, and reproduction in any medium, provided the original author and source are credited.

\begin{abstract}
Objectives: The aim of this study was to compare the maternal and the perinatal outcomes of women with PreGestational Diabetes Mellitus (PGDM) with the outcomes of those with Gestational Diabetes Mellitus (GDM).

Methods: In a retrospective cohort study the maternal and the neonatal outcomes of women with PGDM, were compared to the outcomes of women with GDM. Data were collected for the period of 12 months, from the $1 \mathrm{st}$ of January to the 31st of December 2012, from the labour ward registry. Data compared were; age, parity, mode of delivery, premature delivery, previous history of miscarriage, birth weight, macrosomia, rate of APGAR scores less than 7 at 5 minutes and stillbirth rate. Student $t$ test was used to compare continuous variables and Chi squared was used to compare categorical variables. Multivariate analysis was used to estimate adjusted Odds Ratio (OR).
\end{abstract}

Results: Of the 3413 deliveries during the study period, 3157 fulfilled the inclusion criteria; of those there were a total of 685 deliveries for diabetic women. $569(83.1 \%)$ had GDM and $116(16.9 \%)$ had PGDM. Of those with PGDM, $66(57 \%)$ had T1DM and $50(43 \%)$ had T2DM.

Compared to the GDM group, women with PGDM were more likely to be delivered by caesarean section (CS), adjusted OR $2.6,95 \% \mathrm{Cl}(1.66-4.09)$. The neonates of mothers with PGDM were significantly heavier compared to those of GDM group, p<0.001; and the frequency of macrosomia was more, adjusted OR $3.67,95 \% \mathrm{Cl}(1.75-7.71)$. Mothers with PGDM have increased risk of preterm delivery less than 37gestations weeks, adjusted OR $2.63,95 \%$ $\mathrm{Cl}(1.49-4.70)$. There was no statistically significant difference between the two groups in the rate of APGAR scores less than 7 in 5 minutes or the rate of stillbirth.

Conclusion: PGDM is associated with worse pregnancy outcomes compared to GDM.

Keywords: Gestational diabetes; Pre-gestational diabetes; Cesarean section; Macrosomia; Stillbirth

\section{Introduction}

Pre-Gestational (PGDM) and Gestational (GDM) diabetes mellitus are associated with poor pregnancy outcomes [1]. There is great variation in the prevalence and the impact of PGDM and GDM among different ethnic groups $[2,3]$. Epidemiological studies confirmed that the prevalence of GDM is in direct proportion to the prevalence of type 2 diabetes mellitus (T2DM) [4]. Moreover many pre-diabetic risk conditions, such as obesity and age above 30 years, are associated with both conditions [4] and women who developed GDM are at increased risk of developing T2DM $[5,6]$.

Recent population based studies in Saudi Arabia estimated the prevalence of T2DM to be between $21 \%$ to $24 \%$, which reflects a fivefold increase in the affected population in just over 20 years $[7,8]$. Among the Middle East countries, the Gulf region countries showed the highest prevalence of Diabetes Mellitus (DM); with the Kingdom of Saudi Arabia (KSA) reported the highest prevalence compared to the other Gulf countries. The rapidly increasing prevalence of T2DM has been attributed to the fast changes in lifestyle, dietary habits, and physical activity of the Saudi community associated with the socioeconomic changes and fast urbanization [9]. The World Health Organization predicted that DM prevalence in KSA will increase by $183 \%$ over the 20 years following 2003 [10].

Despite the confirmed high prevalence of DM, only few studies addressed the prevalence and the effect of maternal diabetes on pregnancy outcomes in KSA. Similar to other parts of the world diabetes in pregnancy in KSA is associated with increased maternal age, parity and body mass index [11-13].

The physiological changes of pregnancy cause a state of carbohydrate intolerance. The pregnancy specific hormones such as human placental lactogen and the increased levels of cortisol and prolactin increase the insulin resistance and call for more production of the hormone to maintain normal blood glucose level during pregnancy. Such demand is not met in pregnant diabetic women due to the pathology associated with diabetes.

GDM "is any degree of carbohydrate intolerance that begins or is first recognized during pregnancy" [14]. The hyperglycemia in GDM 
typically appears late in pregnancy, hence the recommended screening time between 24-28 gestation weeks.

The effect of hyperglycemia on the pregnancy outcome varies with the level of maternal blood glucose and the time during pregnancy with uncontrolled hyperglycemia. Hyperglycemia occurring early in pregnancy and during organogenesis, as in the case of uncontrolled T1DM and T2DM, is associated with risk of congenital malformations, macrosomia, stillbirth, birth asphyxia and preterm delivery, while the same complications might appear with GDM but less frequent and less severe due to the late occurrence of the hyperglycemia $[15,16]$.

The aim of this study is to compare the maternal and the perinatal outcomes of women with PGDM with the outcomes of those with GDM.

\section{Methods}

This is a retrospective cohort study to investigate the maternal and the neonatal outcomes of women with PGDM (T1DM and T2DM), and those with GDM who delivered in King Khalid University Hospital (KKUH).

KKUH is an 800 bed tertiary referral center. It has an emergency department 20 operating theaters, an assisted reproduction unit and a cardiac center. The hospital provides free medical care to Saudi Nationals and the staff of King Saud University. The obstetric department provides care for 3000-4000 deliveries per year.

The data were collected for the period of 12 months from the 1st of January to the 31 st of December 2012 from the labour ward registry and missing data were obtained from the maternal medical records. The demographic characteristics and the pregnancy outcomes of the women with GDM and PGDM were compared.

The inclusion criteria for this study were:

1. Singleton pregnancy

2. Gestational age of 24 weeks or more at the time of delivery

3. Availability of documented records of maternal glycemic status (Non-diabetic,

\section{GDM or PGDM)}

The exclusion criteria included:

1. Multiple- pregnancy.

2. Women who were not screened for gestational diabetes during the index pregnancy.

The maternal variables assessed were; age, gravidity, parity, gestation age at delivery, mode of delivery, premature delivery at less than 37 weeks of gestation and previous history of miscarriage. The neonatal outcomes included birth weight, macrosomia (birth weight $\geq 4 \mathrm{~kg}$ ) and rate of APGAR scores less than 7 at 5 minutes and the rate stillbirth.

\section{Screening, diagnosis and treatment of GDM}

All women booked for antenatal care at KKUH are screened using Fasting Blood Glucose (FBG) during their first antenatal visit (first trimester or early second trimester). Values above $5.8 \mathrm{mmol} / \mathrm{l}$ indicate a full Glucose Tolerance Test (GTT). Further screening is carried out between 24-28 gestation weeks for women with risk factor/s for GDM according to the American Diabetes Association (ADA). Oral glucose
(50 g) was administered, regardless of the time of the last meal. Venous plasma glucose was measured $1 \mathrm{~h}$ later. A value of $7.8 \mathrm{mmol} / \mathrm{l}$ $(140 \mathrm{mg} / \mathrm{dl})$ or more indicated the need for a full diagnostic GTT. The diagnosis of GDM is based on the results of a 3-h, 100-g oral glucose tolerance test during pregnancy, interpreted according to the diagnostic criteria of O'Sullivan and Mahan [17]. Definitive diagnosis requires that two or more of the venous plasma glucose concentrations exceed: fasting, $5.3 \mathrm{mmol} / \mathrm{l}(95 \mathrm{mg} / \mathrm{dl})$, one hour, $10.0 \mathrm{mmol} / \mathrm{l}(180 \mathrm{mg} /$ $\mathrm{dl})$, two hours, $8.6 \mathrm{mmol} / \mathrm{l}(155 \mathrm{mg} / \mathrm{dl})$ and three hours, $7.8 \mathrm{mmol} / \mathrm{l}$ $(140 \mathrm{mg} / \mathrm{dl})$.

Once diagnosed, women with GDM follow a specific course of treatment including nutritional therapy and counselling together with antenatal fetal surveillance. Insulin therapy is introduced when nutritional therapy fails to maintain the FBG at $5.8 \mathrm{mmol} / 1$ (105 $\mathrm{mg} / 100 \mathrm{ml})$ and/or the two hours postprandial at $7.8 \mathrm{mmol} / \mathrm{l}(140 \mathrm{mg} /$ dl). Women were considered to have PGDM if they were diagnosed before pregnancy. All women with PGDM received insulin during pregnancy for control of blood glucose. None of the study group received oral hypoglycemic agents during pregnancy.

\section{Statistical analysis}

We compared means using the Student $t$ test for continuous variables after assessing normality distribution of the variables, and Chi squire for categorical variables. Data were analyzed using Statistical Package for the Social Sciences (SPSS), Version 17 (SPSS Inc., Chicago, IL, USA). Odds Ratio (OR) was calculated and P-value of $<0.05$ indicated statistical significance. Multivariate analysis was used to calculate adjusted Odds Ratio (OR), adjustment was performed for potential confounding factors including; maternal age, parity and gestational age. P-value of $<0.05$ indicated statistical significance.

Ethical approval was sought and granted before commencing the study from the institutional ethics review board of Collage of Medicine King Saud University.

\section{Results}

Of the 3413 deliveries during the study period, 3157 deliveries fulfilled the inclusion criteria. From the study population there were a total of 685 deliveries for diabetic women. $569(83.1 \%)$ had GDM and 116 (16.9\%) had PGDM. Of those with PGDM, 66 (57\%) had T1DM and $50(43 \%)$ had T2DM. The prevalence of PGDM in this cohort was $3.7 \%$ and that of GDM was $18 \%$.

The comparison of the maternal demographic characteristics of women with GDM and PGDM are shown in Table 1. Mothers with PGDM were significantly older; had significantly more pregnancies and of significantly higher parity compared to the GDM group. Women with PGDM had worse pregnancy outcomes (Table 2).

They are more likely to be delivered by emergency Cesarean Section (CS), Odds Ratio (OR) 1.94, 95\% Confidence Intervals (CI) (1.14-3.28), $\mathrm{p}=0.008$ or elective CS, OR 3.68, 95\%CI (2.05-6.58), $\mathrm{p}<0.001$. The adjusted OR for delivery by CS (elective and emergency) was 2.6, 95\% CI (1.66-4.09). The frequency of APGAR scores less than 7 in 5 minutes was more among neonates of mothers with PGDM compared to those of GDM, however the difference did not reach statistical significance after adjustment for confounding factors, adjusted OR 2.36, 95\%CI (0.64-8.68), $\mathrm{p}=0.11$. 


\begin{tabular}{|l|l|l|l|}
\hline Characteristic & $\begin{array}{l}\text { Gestational } \\
\text { Diabetes Mellitus } \\
569\end{array}$ & $\begin{array}{l}\text { Pre-gestational } \\
\text { Diabetes Mellitus } \\
116\end{array}$ & P value \\
\hline Maternal age & $32.40 \pm 5.89$ & $34.95 \pm 5.66$ & $<0.001$ \\
\hline Gravidity & $4.92 \pm 3.02$ & $6.43 \pm 3.46$ & $<0.001$ \\
\hline Parity & $3.19 \pm 2.46$ & $4.53 \pm 2.90$ & $<0.001$ \\
\hline $\begin{array}{l}\text { History of previous } \\
\text { miscarriage }\end{array}$ & $237(41.8 \%)$ & $54(46.6 \%)$ & 0.35 \\
\hline
\end{tabular}

The neonates of the mothers with PGDM were significantly heavier when compared to those of GDM group, $\mathrm{p}<0.001$; the frequency of macrosomia was more than threefold among PGDM group compared to GDM group, adjusted OR 3.67, 95\% CI (1.75-7.71), $\mathrm{p}=0.02$. Mothers with PGDM have increased estimated risk of preterm delivery less than 37 gestation weeks, adjusted OR 2.63, 95\%CI (1.49-4.70), p 0.003 . Although there was twofold increase in frequency of stillbirth among women with PGDM compared to the GDM group the difference was not statistically significant, adjusted OR $1.96,95 \% \mathrm{CI}$ (0.45-8.50), $\mathrm{p}=0.050$.

Table 1: Maternal demographic characteristics

\begin{tabular}{|c|c|c|c|c|}
\hline \multirow{2}{*}{ Characteristic } & Gestational Diabetes Mellitus & $\begin{array}{l}\text { Pre-gestational Diabetes } \\
\text { Mellitus }\end{array}$ & \multirow{2}{*}{ OR (95\%Cl) } & §Adjusted OR \\
\hline & 569 & 116 & & $(95 \% \mathrm{Cl})$ \\
\hline Emergency $\mathrm{C} / \mathrm{S}$ & $98(17.3 \%)$ & $28(24.1 \%)$ & $1.94(1.14-3.28)$ & \\
\hline Elective C/S & $48(8.5 \%)$ & $26(22.4 \%)$ & $3.68(2.05-6.58)$ & \\
\hline C/S (elective and emergency) & $148(26.0 \%)$ & $54(46.6 \%)$ & $2.47(1.64-3.73)^{*}$ & $2.6(1.66-4.09)^{*}$ \\
\hline APGAR scores at 5 minutes $<7$ & $6(1.1 \%)$ & $5(4.3 \%)$ & $4.21(1.09-15.9)$ & $2.36(0.64-8.68)$ \\
\hline Birth weight & $3197.60 \pm 556.67$ & $3227.34 \pm 804.88^{*}$ & & \\
\hline Macrosomia & $30(5.3 \%)$ & $13(11.2 \%)$ & $2.26(1.08-4.68)$ & $3.67(1.75-7.71)^{\star}$ \\
\hline Stillbirth & $5(0.9 \%)$ & $4(3.4 \%)$ & $4.01(0.89-17.52)$ & $1.96(0.45-8.50)$ \\
\hline Delivery $<37$ weeks & $48(8.5 \%)$ & $21(18.1 \%)$ & $2.39(1.32-4.31)$ & $2.63(1.49-4.70)^{\star}$ \\
\hline
\end{tabular}

Table 2: Comparison of maternal and perinatal outcomes between women with gestational and pre-gestational diabetes mellitus; OR= Odds Ratio, $\mathrm{CI}=$ Confidence intervals, $\mathrm{C} / \mathrm{S}=$ Cesarean section; $\$$ Adjustment for maternal age, parity and gestational age ${ }^{*} \mathrm{p}$-value $<0.05$

\section{Discussion}

The results of this study showed that the studied cohort has high prevalence of both PGDM and GDM. It demonstrated that mothers with PGDM had worse pregnancy outcomes compared to those with GDM. They were more likely to be delivered by CS, had babies who were on average heavier than those born to GDM mothers and more likely to be macrosomic. Newborns of mothers with PGDM were at increased risk of having low APGAR scores at birth, being preterm or born as stillbirth.

The high prevalence of the PGDM and GDM in this cohort is reflection of the high prevalence of T2DM in the Saudi community demonstrated among pregnant women with detrimental consequences on the mothers and their offspring.

In this study the demographic characteristics of the group with GDM were significantly different from the PGDM group. Similar to other studies mothers with PGDM were older and of higher parity, however contrary to our expectations there was insignificant difference in the previous miscarriage rate between the two groups $[15,16]$. This result might be explained with the presence of women with undiagnosed T2DM among the GDM group. Our results confirmed the findings of other investigators about the worse perinatal and maternal outcomes of pregnancies complicated by PGDM compared with the outcomes of pregnancies complicated by GDM $[1,15,16,18]$. It is worse mentioning that, in a previous study from the same hospital, the outcomes of pregnancies complicated with GDM were significantly worse than those of non-diabetic women; compared with the non-diabetic women, women with GDM were 1.7 times more likely to be delivered by CS, the neonates of the mothers with GDM were significantly heavier and almost twice as likely to be macrosomic [19].

Many factors might have influenced the significant difference in the outcomes between these two conditions such as the prolonged exposure of the fetus to maternal hyperglycemia in the case of PGDM with the resultant prolonged fetal hyper-insulinemia and increased Cpeptide level and thus more severe effect on fetal weight gain, macrosomia and the related complications such as CS delivery [20,21]. Moreover prolonged hyperglycemia in PGDM affects the placental vascular bed and can results in increased risk of stillbirth, antenatal and intra-partum asphyxia and hence the low APGAR scores at birth noted in this study. Such observation could have been supported further if enough data were available to investigate the effect of duration of PGDM on the birth weight and the stillbirth rate [22].

The stillbirth rate for women with PGDM reported in this study $(34 / 1000)$ (Table 2), is high compared to rates reported by other investigators $(9.7-26.8 / 1000)[23,24]$, however, the rate of preterm delivery less than 37 gestation weeks, was comparable to the rate reported from a population-based study in Australia and lower than those reported from other studies in England and the Netherlands $[23,25]$. Similarly the rate of macrosomic infants of women with 
PGDM in this study was lower than that reported by other investigators $[1,23]$. This variation in the rates of different outcomes might be explained by the access, utilization and availability of services provided to women with PGDM. Due to the high prevalence of T2DM in the Saudi community, the management of pregnant diabetic women is part of the routine obstetrics practice in secondary and tertiary health care settings, therefore all maternity units, including KKUH, have established their local protocols for antenatal, perinatal and postnatal management of diabetic pregnant women and their infants [7]. The availability and the free access to maternity care during pregnancy and delivery and the acquaintance with the management of diabetic pregnancy by the obstetricians, might explain the lower rate of preterm delivery and macrosomia observed in this study as compared to the published literature. Conversely preconception care, which is effective in improving the perinatal mortality rate in women with PGDM was not an integral part of the health services provided for women with T1DM and T2DM in the hospital during the study period, consequently lack of awareness from the women part about the importance of glycemic control in the peri-conception period and lack of use of folic acid during the preconception period, might explain the high rate of stillbirth in this study compared to similar studies [26].

\section{Implication to practice}

This study has provided valuable information about the complications associated with diabetic pregnancy. Given the high prevalence of both conditions in the cohort, the study proved that diabetes in pregnancy is a major health problem in the maternity unit in KKUH which warrants standardization of screening and treatment.

\section{Implication to research}

The results of this study showed that more than $20 \%$ of women giving birth during the study period in KKUH, have either GDM or PGDM and at risk of adverse pregnancy outcomes. Such high prevalence in one health institution calls for a national prospective survey to estimate the prevalence of GDM and PGDM in the different regions of the Kingdom and to evaluate the national impact of diabetes in pregnancy on the maternal and perinatal outcomes. We are aware of the limitation of this study including the retrospective nature of the design and the lack of data on important outcomes such as preeclampsia, congenital malformations and perinatal mortality. Other limitations include, lack of data on potential confounders for the outcomes such as the gender of the newborn and maternal weight. We cannot exclude the possibility of misclassification of the study population because the obstetric department in KKUH adopts selective screening for GDM based on risk factors. In addition a single center experience might hinder the extrapolation of the results to other regions of the Kingdom.

\section{Conclusion}

PGDM is associated with worse maternal and fetal outcomes compared to GDM however the outcomes of PGDM are comparable to those reported from other countries except in the stillbirth rate which could be improved by establishment of the services of preconception care.

\section{Authors' Contribution}

Hayfaa Wahabi conceived the idea for the study; she supervised the data collection and analysis and she wrote the final manuscript. Samia
Esmaeil managed the data. Amel Fayed analyzed the data and extracted the results. All authors read the final draft of the manuscript and approved it.

\section{Acknowledgement}

We acknowledge the help we received from Ms. Bella Magnaye in collecting and tabulating the data.

\section{References}

1. Shand AW, Bell JC, McElduff A, Morris J, Roberts CL (2008) Outcomes of pregnancies in women with pre-gestational diabetes mellitus and gestational diabetes mellitus; a population-based study in New South Wales, Australia, 1998-2002. Diabet Med 25: 708-715.

2. Verheijen EC, Critchley JA, Whitelaw DC, Tuffnell DJ (2005) Outcomes of pregnancies in women with pre-existing type 1 or type 2 diabetes, in an ethnically mixed population. BJOG 112: 1500-1503.

3. Savitz DA, Janevic TM, Engel SM, Kaufman JS, Herring AH (2008) Ethnicity and gestational diabetes in New York City, 1995-2003. BJOG 115: 969-978.

4. Ben-Haroush A, Yogev Y, Hod M (2004) Epidemiology of gestational diabetes mellitus and its association with Type 2 diabetes. Diabet Med 21: 103-113.

5. Jang HC (2011) Gestational diabetes in Korea: incidence and risk factors of diabetes in women with previous gestational diabetes. Diabetes Metab J 35: 1-7.

6. Albareda M, Caballero A, Badell G, Piquer S, Ortiz A, et al. (2003) Diabetes and abnormal glucose tolerance in women with previous gestational diabetes. Diabetes Care 26: 1199-1205.

7. Al-Daghri NM, Al-Attas OS, Alokail MS, Alkharfy KM, Yousef M, et al. (2011) Diabetes mellitus type 2 and other chronic non-communicable diseases in the central region, Saudi Arabia (Riyadh cohort 2): a decade of an epidemic. BMC Med 9: 76.

8. Fatani HH, Mira SA, el-Zubier AG (1987) Prevalence of diabetes mellitus in rural Saudi Arabia. Diabetes Care 10: 180-183.

9. Majeed A, El-Sayed AA2, Khoja T3, Alshamsan R4, Millett C5, et al. (2014) Diabetes in the Middle-East and North Africa: an update. Diabetes Res Clin Pract 103: 218-222.

10. Khandekar R, Al Lawatii J, Mohammed AJ, Al Raisi A (2003) Diabetic retinopathy in Oman: a hospital based study. Br J Ophthalmol 87: 1061-1064.

11. Al-Rowaily MA, Abolfotouh MA (2010) Predictors of gestational diabetes mellitus in a high-parity community in Saudi Arabia. East Mediterr Health J 16: 636-641.

12. El-Gilany AH, Hammad S (2010) Body mass index and obstetric outcomes in pregnant in Saudi Arabia: a prospective cohort study. Ann Saudi Med 30: 376-380.

13. Meher-un-nisa, Muhammad Aslam, Salah Rosdy Ahmed, Maamon Rajab, and Lina Kattea (2011) Impact of obesity on fetomaternal outcome in pregnant saudi females. Int J Health Sci (Qassim ) 5: 40-41.

14. American Diabetes Association (2010) Diagnosis and classification of diabetes mellitus. Diabetes Care 33 Suppl 1: S62-69.

15. Barakat MN, Youssef RM, Al-Lawati JA (2010) Pregnancy outcomes of diabetic women: charting Oman's progress towards the goals of the Saint Vincent Declaration. Ann Saudi Med 30: 265-270.

16. El Mallah KO, Narchi H, Kulaylat NA, Shaban MS (1997) Gestational and pre-gestational diabetes: comparison of maternal and fetal characteristics and outcome. Int J Gynaecol Obstet 58: 203-209.

17. O'sullivan Jb, Mahan Cm (1964) Criteria For The Oral Glucose Tolerance Test In Pregnancy. Diabetes 13: 278-285.

18. Ray JG, Vermeulen MJ, Shapiro JL, Kenshole AB (2001) Maternal and neonatal outcomes in pregestational and gestational diabetes mellitus, and the influence of maternal obesity and weight gain: the DEPOSIT 
Citation: Wahabi HA, Fayed A, Esmaeil SA (2014) Maternal and Perinatal Outcomes of Pregnancies Complicated with Pre-gestational and Gestational Diabetes Mellitus in Saudi Arabia. J Diabetes Metab 5: 399. doi:10.4172/2155-6156.1000399

Page 5 of 5

study. Diabetes Endocrine Pregnancy Outcome Study in Toronto. QJM 94: 347-356.

19. Wahabi HA, Esmaeil SA, Fayed A, Alzeidan RA (2013) Gestational diabetes mellitus: maternal and perinatal outcomes in King Khalid University Hospital, Saudi Arabia. J Egypt Public Health Assoc 88: 104-108.

20. HAPO Study Cooperative Research Group1, Metzger BE, Lowe LP, Dyer AR, Trimble ER, et al. (2008) Hyperglycemia and adverse pregnancy outcomes. N Engl J Med 358: 1991-2002.

21. Nachum Z, Ben-Shlomo I, Weiner E, Shalev E (1999) Twice daily versus four times daily insulin dose regimens for diabetes in pregnancy: randomised controlled trial. BMJ 319: 1223-1227.

22. Rackham O, Paize F, Weindling AM (2009) Cause of death in infants of women with pregestational diabetes mellitus and the relationship with glycemic control. Postgrad Med 121: 26-32.
23. Confidential Enquiry into Maternal and Child Health (CEMACH). Pregnancy in Women with type 1 and type 2 Diabetes in 2002-2003.England Wales and Northern Ireland. London: CEMACH; 2005.

24. Yang J, Cummings EA, O'connell C, Jangaard K (2006) Fetal and neonatal outcomes of diabetic pregnancies. Obstet Gynecol 108: 644-650.

25. Evers IM, de Valk HW, Visser GH (2004) Risk of complications of pregnancy in women with type 1 diabetes: nationwide prospective study in the Netherlands. BMJ 328: 915.

26. Wahabi HA, Alzeidan RA, Bawazeer GA, Alansari LA, Esmaeil SA (2010) Preconception care for diabetic women for improving maternal and fetal outcomes: a systematic review and meta-analysis. BMC Pregnancy Childbirth 10: 63 . 